\title{
Hydrogen/Deuterium Adsorption and Absorption Properties On and In Palladium Using a Combined Plane Wave and Localized Basis Set Method
}

\author{
Hiroki Sakagami ${ }^{1}$, Masanori Tachikawa ${ }^{2}$, and Takayoshi Ishimoto ${ }^{1}$ \\ ${ }^{1}$ Yokohama City University \\ ${ }^{2}$ Yokohama-City University
}

May 5, 2020

\begin{abstract}
Detailed information on the H/D isotope effects for adsorption on the surface and absorption in the bulk is important for understanding the nuclear quantum effect. To achieve this purpose, we developed a new theoretical approach, namely, the combined plane wave and localized basis set (CPLB) method. By using the multi-component quantum chemical method, which takes into account the quantum effect of proton or deuteron, with localized part in CPLB method, direct analysis of H/D isotope effect about adsorption and absorption is achieved. In this study, we performed a theoretical investigation of the H/D isotope effects for adsorption on a $\mathrm{Pd}(111)$ surface and absorption in bulk Pd. We clearly showed H/D isotope effect on geometry during adsorption and absorption. Our developed CPLB approach is a powerful tool for analyzing the quantum nature of H/D in surface, bulk, and inhomogeneous systems.
\end{abstract}

\section{Hosted file}

IJQC-Ishimoto.docx available at https://authorea.com/users/301846/articles/431736-hydrogendeuterium-adsorption-and-absorption-properties-on-and-in-palladium-using-a-combinedplane-wave-and-localized-basis-set-method 